\title{
Introduction to the contents of issue 51:3
}

\author{
Axel Ruhe
}

Published online: 25 August 2011

(C) Springer Science + Business Media B.V. 2011

In this issue of BIT we continue to report from our part of the scientific field. We have several studies of direct methods to follow the solution of a dynamical system forward in time and some studies of inverse problems, where we have observations, and want to find the system of which these observations is a solution. We have some studies of specific applied problems, but today few studies of implementation aspects of numerical algorithms.

These are the papers that make up BIT 51:3:

Lehel Banjai and Christian Lubich discuss methods to evaluate integral transforms with convolution kernels. The algorithms are related to those for initial value problems for ordinary differential equations. Stability considerations lead to a preference for implicit Runge Kutta algorithms, and error bounds are derived for evaluation of Laplace transforms with kernels that are polynomially bounded in a half plane.

Sergiy Borodachov and Tanya Sorokina use polynomial splines in $d$ dimensions, to recover a function, whose values and first derivatives are known in a set of points. The condition is that the function has bounded second derivatives.

Christine Böckmann and Athassawat Kammanee study algorithms to reconstruct the potential of a non symmetric Sturm Liouville problem from some knowledge of the eigenvalues and eigenfunctions. The algorithms used are the Numerov method to solve the ODE system and Broyden minimization to find the parameters.

Kristian Debrabant and Anne Kvarn $\phi$ analyze convergence of implicit Taylor series methods for stochastic differential equations. They use B-series, as introduced by Kevin and Pamela Burrage, for the iterates of simple and Newton iterations to

\footnotetext{
A. Ruhe ( $₫)$

School of Computer Science and Communications (CSC), Royal Institute of Technology (KTH), 10044 Stockholm, Sweden

e-mail: ruhe@kth.se
} 
analyze both strong and weak convergence. The behavior of these Taylor methods is similar to Runge Kutta methods, but there are interesting differences.

Luca Dieci, Cinzia Elia, and Erik Van Vleck study algorithms to establish whether a linear dynamic system has exponential dichotomy, that means that the space of initial values can be decomposed into one forward stable and one backward stable part. The algorithms are based on QR or SVD decompositions of a fundamental matrix solution.

Etienne Emmrich studies time discontinuous Galerkin methods for nonlinear evolution equations. Conditions for convergence of the approximations to a weak solution of the original problem are established. Bounds of stability and data error estimates are derived.

Hui Feng, Xiaoping Zhang, and Jin Li solve a hypersingular integral equation over the unit circle with a variant of an iterated midpoint rule. They describe a collocation method on these midpoints to compute a numerical solution.

Sebastian Franz studies the convergence of a streamline diffusion finite element method, applied to a singularly perturbed convection diffusion problem with exponential and characteristic boundary layers. A layer adapted mesh is used.

Tomas Johnson and Warwick Tucker study the Abel integrals used to establish the number of limit cycles of a perturbed Hamiltonian system. Methods from interval analysis are used to compute rigorous bounds.

Esther Klann, Ronny Ramlau, and Lothar Reichel solve a Fredholm integral equation of the first kind, an ill posed problem. They describe a cascadic multilevel iteration using a sequence of wavelet bases. At each level, starting on the coarsest grid, conjugate gradient is run for a few steps on the unregularized problem until a discrepancy criterion signals termination. This stopping gives regularization on each level.

Dimitrios Noutsos and Paris Vassalos describe a preconditioned iteration for a system with a symmetric positive definite ill-conditioned block Toeplitz matrix with Toeplitz blocks. The solution of the component systems gives a preconditioner for a conjugate gradient iteration for the whole system.

Jelena Popovic and Olof Runborg study the high frequency one dimension Helmholtz equation with varying speed of wave propagation. The Helmholtz equation is split into one-way wave equations which are solved iteratively, sweeping back and forth over the domain. In regions where the source is zero, a geometrical optics approximation is used.

Sara Remogna studies spline approximation in a three dimensional space partitioned into tetrahedrons. It is shown that quasi interpolation by a trivariate $C 2$ quartic box spline can give approximation order 4 .

Welcome to another issue of BIT!

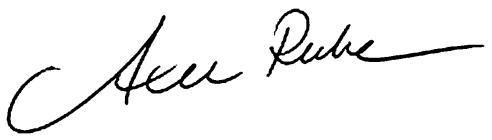

Axel Ruhe 